\title{
Identifying Optimal Portfolios of Resilient Network Investments Against Natural Hazards, With Applications to Earthquakes
}

DOI:

10.1109/TPWRS.2019.2945316

\section{Document Version}

Accepted author manuscript

Link to publication record in Manchester Research Explorer

Citation for published version (APA):

Lagos, T., Moreno, R., Navarro Espinosa, A., Panteli, M., Sacaan, R., Ordonez, F., Rudnick, H., \& Mancarella, P. (2019). Identifying Optimal Portfolios of Resilient Network Investments Against Natural Hazards, With Applications to Earthquakes. I E E E Transactions on Power Systems. https://doi.org/10.1109/TPWRS.2019.2945316

\section{Published in:}

I E E E Transactions on Power Systems

\section{Citing this paper}

Please note that where the full-text provided on Manchester Research Explorer is the Author Accepted Manuscript or Proof version this may differ from the final Published version. If citing, it is advised that you check and use the publisher's definitive version.

\section{General rights}

Copyright and moral rights for the publications made accessible in the Research Explorer are retained by the authors and/or other copyright owners and it is a condition of accessing publications that users recognise and abide by the legal requirements associated with these rights.

\section{Takedown policy}

If you believe that this document breaches copyright please refer to the University of Manchester's Takedown Procedures [http://man.ac.uk/04Y6Bo] or contact uml.scholarlycommunications@manchester.ac.uk providing relevant details, so we can investigate your claim.

\section{OPEN ACCESS}




\begin{abstract}
Although extreme natural disasters have occurred all over the world throughout history, power systems planners do not usually recognize them within network investment methodologies. Moreover, planners had historically focused on reliability approaches based on average (rather than risk) performance indicators, undermining the effects of high impact and low probability events on investment decisions. To move towards a resilience centred approach, we propose a practical framework that can be used to identify network investments that offer the highest level of hedge against risks caused by natural hazards. In a first level, our framework proposes network enhancements and, in a second level, uses a simulation to evaluate the resilience level improvements associated with the network investment propositions. The simulator includes 4 phases: threat characterization, vulnerability of systems components, system response, and system restoration, which are simulated in a sequential Monte Carlo fashion. We use this modeling framework to find optimal portfolio solutions for resilient network enhancements. Through several case studies with applications to earthquakes, we distinguish the fundamental differences between reliability- and resilience-driven enhancements, and demonstrate the advantages of combining transmission investments with installation of backup distributed generation.
\end{abstract}

Index Terms-Resilient network planning, natural hazards, earthquakes, resilience, resiliency, reliability.

\section{A. Motivation}

\section{INTRODUCTION}

Major natural hazards present severe negative impacts on countries' economies [1], in particular due to their effect on critical infrastructure such as electricity networks. Indeed, the effects of long electricity blackouts have demonstrated severe impacts not only on economic activities but also on social stability and security [2]. Hence, there is a growing consensus across the globe to increase levels of power system resilience, which is reflected in recent research and in new governmental policies aimed at mitigating the negative effects of natural hazards. For instance, the Chilean electricity regulator issued a new transmission law in 2016 that requires planning electricity transmission networks in a resilient fashion by "hedging risks against natural disasters and extreme hydrological conditions" [3].

Many countries are significantly exposed to various types of natural hazards. For example, in the ring of fire region (i.e. countries located at the edge of the pacific ocean), earthquakes represent a significant threat, being responsible for more than half of the economic losses and human fatalities among all natural disasters in countries like China and Chile [4], [5]. Hence, protecting critical infrastructure and, in particular, electricity networks against earthquakes will reduce exposure to grave problems faced in earthquake prone areas of the world.

\section{B. Literature review}

Power system resilience can be defined as the ability of a power system to withstand high impact and low probability (HILP) catastrophic phenomena (such as extreme weather events, natural disasters and man-made attacks), recover quickly from such disruptive events and, in the longer term, adapt its operation and infrastructure to prevent or mitigate the impacts of similar events in the future [6], [7]. Recently, an IEEE task force [8] has provided the following definition of resilience: "The ability to withstand and reduce the magnitude and/or duration of disruptive events, which includes the capability to anticipate, absorb, adapt to, and/or rapidly recover from such an event".

In the context of definitions and general frameworks, in [9] and [10] a conceptual framework of power system resilience is presented and discussed along with the key measures that can be undertaken to improve network resilience. In [11] four indices or metrics are introduced to measure network resilience from different perspectives, including fragility, survival and restoration. In [12] the concept of the multiphase resilience trapezoid is proposed, presenting novel metrics to quantify resilience in each stage of the collapse-recovery process. Additionally, [13] proposes a risk aversion framework that can be used to operate and design networks in a more resilient fashion, reducing exposure to adverse weather conditions and extreme natural disasters.

In the context of resilience assessment, [14] proposed four phases to assess power system resilience: threat characterization, vulnerability assessment of system components, system response and system restoration. Similarly, reference [15] proposes a resilience assessment method divided into three stages: hardness before disasters, resistance during disasters and capacity of restoration after disasters. Besides this multitemporal perspective, in [16] a fragility model of the transmission system is developed that also emphasizes the multiregional component within the probabilistic methodology proposed for resilience assessment. In [17] a framework for assessing resilience against seismic events is described along with the evaluation of various mitigating investment strategies, applied on a real electricity grid in Chile.

In the context of improving system resilience, previous literature (such as [9], [13], [17]-[23]) has proposed several 
operational and investment measures. Hence, in the optimal planning context, there are different models of investment planning/expansion like those introduced in [20], [24], [25]. Reference [24] proposes a transmission expansion problem using a multi-level mixed integer programming (MIP) model to make investment decisions under terrorist threats. Reference [20] proposes a two-stage stochastic program and its corresponding solution algorithm to optimize investments that improve resilience against earthquakes. Another twostage stochastic optimization model is also proposed in [25], confirming that stochastic optimization approaches are a promising tool for appropriate resilient decision making. Hence our work presented here also proposes a two-stage stochastic framework to identify network enhancements to improve resilience against earthquakes, using an Optimization via Simulation $(\mathrm{OvS})$ solution approach. This allows to capture a very high level of detail and complexity in the simulation stage, including a comprehensive set of operational constraints and the sequential process of disconnection and reconnection of loads, which is key in evaluating the dynamics of resilience and has not been properly addressed in existing relevant works in network investment planning.

\section{Contributions}

This paper presents three main contributions. First, we provide a mathematical framework to identify (nearly ${ }^{1}$ ) optimal resilient network investments, which are optimized by testing the performance of a comprehensive set of system enhancements against a series of outages originated by natural hazards as illustrated in Fig. 1. Importantly, this framework is based on an OvS approach that provides a more realistic representation of electricity network behavior, including disconnections and reconnections of loads and system infrastructures. Second, and following existing literature [12], we select optimal portfolios of network investments while attempting to improve different aspects of system resilience represented through different metrics. Hence, in the proposed framework, the resilience metric being optimized can be freely chosen.

Third, through various studies we demonstrate the differences between reliable and resilient network enhancements, where in the former we do not explicitly model the occurrence of high impact exogenous natural hazards, focusing on reliability improvements across a comprehensive set of outages (not only those driven by HILP events), generally dominated by low impact and high probability failures ${ }^{2}$. Instead, in resilience studies, we focus on improving performance under HILP events. In this vein, we demonstrate that hardening

\footnotetext{
${ }^{1}$ We refer to the term "nearly optimal" throughout this paper as the optimization is undertaken through simulations and there is no guarantee of global optimality. As we will demonstrate later in Section III-C, though, our results are indeed truly optimal for the analyzed cases.

${ }^{2}$ Under the reliability approach, one may argue that high impact exogenous events can be incorporated within failure probability values, along with all other events. Furthermore, the underlying process behind the realization of system contingencies is traditionally modeled in reliability studies by using marginal probabilities of failure, which can be obtained by marginalizing (or "averaging") probability values across a number of operating conditions with and without the effects of exogenous events. However, as high impact exogenous events are extremely rare, it is likely that these will present a very limited impact on the marginal probability values used under the reliability approach.
}

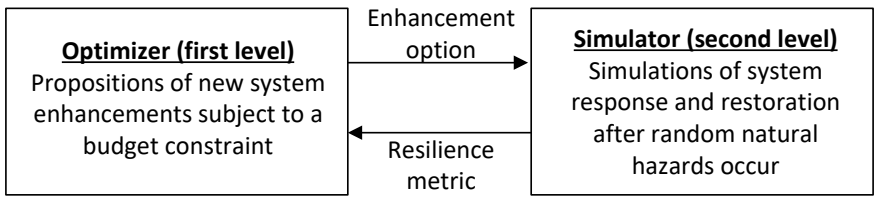

Fig. 1. OvS approach for resilient network investments.

substations can significantly contribute towards enhancing resilience to high impact exogenous natural hazards. This is in contrast to traditional reliability approaches that favor network redundancy, i.e. new transmission lines. We also demonstrate the benefits of portfolio solutions containing a mixture of new network infrastructure (e.g. lines and transformers), substation hardenings and backup distributed generation (DG).

Finally, note that although we illustrate the proposed modeling framework on earthquakes, this framework can be applied on other hazards too.

\section{Paper structure}

This paper is structured as follows. Section II presents the modelling framework to determine resilient network enhancements based on an OvS approach. Section III presents the case studies and shows our results and discussions. Finally, Section IV concludes.

\section{MODELING FRAMEWORK FOR RESILIENT NETWORK INVESTMENTS}

\section{A. General overview}

We use an OvS approach to determine the (nearly) optimal portfolio of resilient network investments. This framework exploits the bi-level structure of the network investment problem, whereby, in a second level (namely, the simulator), network operation is simulated over a network infrastructure that is determined in a first level (namely, the optimizer). As the focus of this application is on resilience, we are particularly interested in simulating system operation under a number of (simultaneous) system outages that are caused by natural hazards. Hence, consequences of natural hazards are simulated under various network enhancement solutions, proposed by the optimizer. Optimizer and simulator iterates until a (nearly) optimal, resilient network enhancement proposition is found, as illustrated in Fig. 1.

For the simulator, we use a toolbox with a number of models to simulate the hazard (including its occurrence and spatio-temporal propagation profile) and its impacts on the electricity network (i.e. the system response and restoration). Following the nomenclature in [14], we formally call these steps as follows: 1) Threat characterization, 2) Vulnerability of system's components, 3) System response, and 4) System restoration. These steps $1-4$ are run sequentially within the simulator in order to obtain a full simulation of the electricity network before, during and after the natural hazard occurs.

The simulator features three layers of uncertainty modelling. The first one within the threat characterization stage, where we need to consider various scenarios of natural hazards, attempting to capture their varieties in terms of locations, magnitudes, etc. The second layer is related to the vulnerability of system components, which is parameterized in a set of failure rates through component fragility curves [26], which 
are hazard dependent. Given these failure rates (i.e. outage probabilities), we determine a number of scenarios representing different network outages originated by each natural hazard. Finally, the third layer of uncertainty corresponds to the repair rate of network components, whose times are assumed to be exponentially distributed, following [27]. For the three uncertainty layers we use a Monte Carlo method to simulate different scenarios of hazards, the corresponding network outages, and their repairs.

For the optimization part, we use OvS techniques, in particular an algorithm named Industrial Strength Compass (ISC), originally published in [28], which determines the (nearly) optimal portfolio of network enhancements based on a series of simulations. Such enhancements may include new network equipment (lines, transformers, etc.), substation hardenings and reinforcements, etc. A key point to highlight is that some of the uncertainty is decision dependant, making the objective function to be optimized non-linear. For instance, if a substation is hardened, its failure rates become smaller (note that in traditional optimization models used to plan network investments with probabilistic outages like in [13], [29], the values of outage probabilities are constant, representing a set of parameters for the optimization model and thus allowing us to formulate a fully linear model). While stochastic mathematical programs have difficulty considering decision dependent probabilities, OvS techniques represent a pragmatic method to address this problem.

\section{B. The simulator}

1) Threat characterization: We use a probabilistic approach to model the magnitude and the spatio-temporal profile of the hazard, specifically earthquakes, in our case studies. Particularly, we run a Monte Carlo model that generates different scenarios for a chosen hazard. In the case of earthquakes, we need to model, firstly, their magnitudes and locations and, secondly, their (spatial) attenuation profile. For the magnitudes, we use the Gutenberg-Richter number-size exponential distribution of earthquake magnitudes [30]. For the locations, we could use either historical data or more advanced platebased modeling to identify those locations that are more likely to be affected by an earthquake [31]. For the attenuation, we use the model proposed by Boroschek [32] (suitable for Chile), but this can be changed according to the specific application of the model. Boroschek proposes that peak ground acceleration (PGA) attenuation at any position $r$ from the earthquake's epicenter follows (1).

$$
\begin{gathered}
\log _{10}(P G A(r, h, M))=-1.55+0.26 M \\
+0.01 h-0.01 R-(1.52-0.10 M) \log _{10}(R)
\end{gathered}
$$

Where $M$ is the earthquake's magnitude in the GutenbergRichter scale. Given the hypocenter $(e x, e y, h)$, then $r=$ $\sqrt{(e x-x)^{2}+(e y-y)^{2}}$ and $R$ is $\sqrt{r^{2}+\left(0.07 \cdot 10^{0.36 \cdot M}\right)^{2}}$. The results is on units of $[g]$, the gravity acceleration constant.

2) Vulnerability of systems components: Here, we determine the failure rates or probabilities of outages of system components, conditional to the occurrence of a natural hazard, specifically fragility curves give the probability distribution over two or more damage states as a function of given spatial

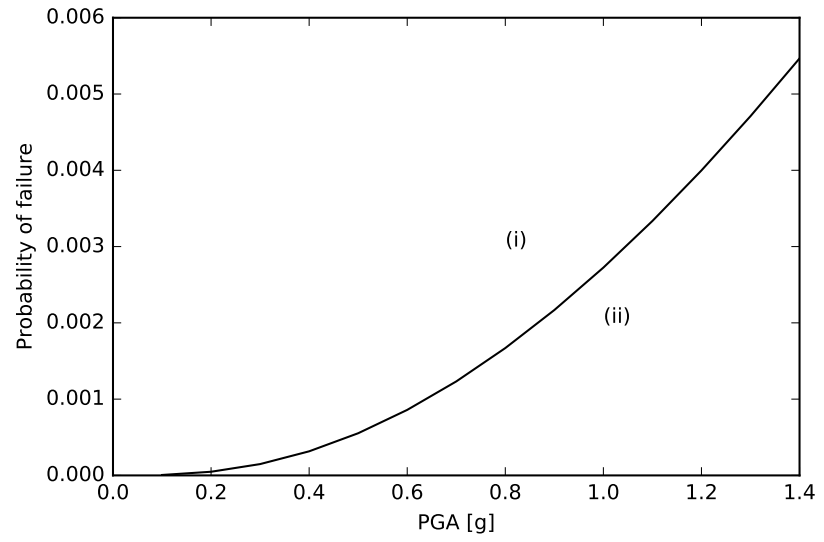

Fig. 2. Tower's fragility curve with (i) functioning and (ii) outaged state.

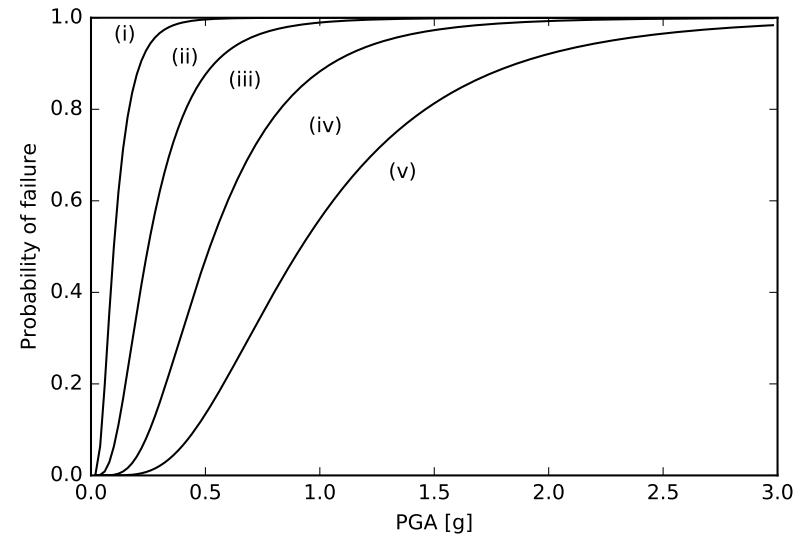

Fig. 3. Generator's fragility curves with 5 states: (i) no damage, (ii) minor, (iii) moderate, (iv) extensive, and (v) complete damage.

PGA at the location due to the earthquake, obtained from (1). To do so, we use the fragility curves in [26] that determine the failure probability as a function of the PGA at its location for different network components. In particular, we consider fragility curves for the following infrastructure:

Transmission towers: that can present only two states after an earthquake occurs: (i) fully functioning or (ii) outaged. In this case, we need a single fragility curve to represent the on/off states as explained in [26]. We consider that a transmission line fails if, at least, one of its carrying towers fails. This is shown in Fig. 2.

Generating units: that can present various states after an earthquake occurs. We use the approach in [26] with 5 states: (i) fully functioning (no damage), (ii) minor, (iii) moderate, (iv) extensive, and (v) complete damage. In this case, we need 4 fragility curves to represent 5 states. The generation available capacities associated with the (i)-(v) states are $100 \%, 90 \%$, $60 \%, 30 \%$, and 0\%, respectively. This is shown in Fig. 3.

Substations: that can present various states after an earthquake occurs. We use the approach in [26] with 5 states: (i) fully functioning (no damage), (ii) minor, (iii) moderate, (iv) extensive, and (v) complete damage. In this case, we need 4 fragility curves to represent 5 states. In [26], there are two sets of fragility curves, one to represent substations that have 


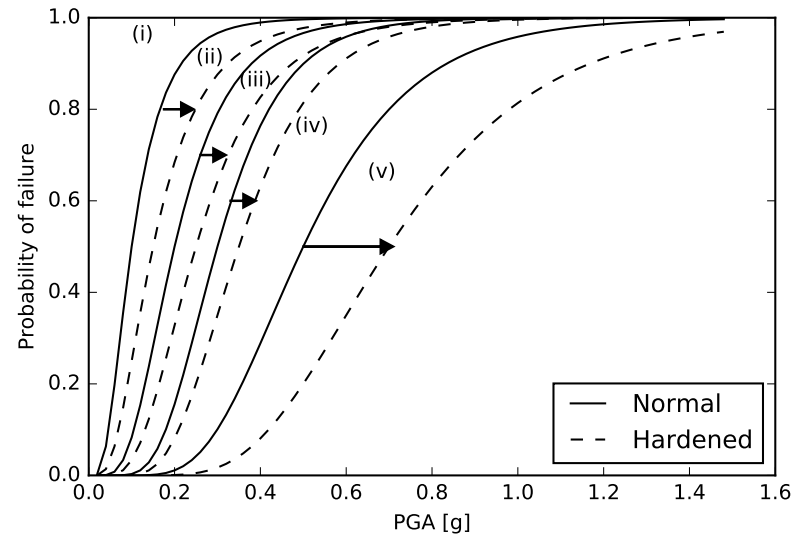

Fig. 4. Substation's fragility curves with 5 states: (i) no damage, (ii) minor, (iii) moderate, (iv) extensive, and (v) complete damage, indicating curves for the normal and hardened cases.

been hardened and another set to represent substations that have not. We use both sets as hardening is a decision variable. The substation available capacities associated with the (i)-(v) states are $100 \%, 90 \%, 60 \%, 30 \%$, and $0 \%$. This is shown in Fig. 4, where the arrows indicate the shift of the curves due to hardening measures. As in dispatch models (needed to determine the system response and restoration and explained in the next subsection) substations do not explicitly feature a capacity value, the aforementioned derate on a substation capacity is translated into a derate applied on the capacity value of all elements connected to that substation (lines, generating units, etc.).

After we have determined the outage/state probability of every network component, we run a Monte Carlo simulation to determine various scenarios where network components are outaged/derated. For these network conditions (where each may present several simultaneous outages), we model the system response as explained next.

3) System response and restoration: We determine the system response and restoration through two well-established power system models: a (deterministic) unit commitment (UC) and a post-contingency dispatch (PCD), where the latter is subject to the results obtained by the former. We model system operation during a time horizon that is meaningful for the particular hazard being studied (e.g. experience in Chile suggests five days for large earthquakes). Within the targeted time horizon, we firstly run the unit commitment model (in particular, we use the formulation in [33]) in order to define the intact system condition, i.e. scheduled/planned unit commitment. After such intact system operation has been determined, we then generate scenarios of natural hazards with their corresponding system outages, and model the system response considering optimal corrective actions by means of the post-contingency dispatch model. In effect, this dispatch model is used for undertaking corrective actions right after the outages occur (by using available reserves and respecting all units' constraints, e.g. ramp rate limits, minimum output limits, etc.) and optimize the operation during the remaining period (e.g. a week after the earthquake occurs in an hourly fashion). This dispatch model can switch on additional units that were not affected by the earthquake and were not initially participating in the scheduled/planned commitment. This model also determines the volumes and locations of energy not supplied (ENS), which (due to its high cost) represent the last resort measure to balance the system after an outage occurs. Meanwhile, outaged system infrastructure is being repaired following an exponential distribution with a particular repair rate per network component. Of particular interest is the energy balance equation (2) of the dispatch model where corrective actions, in particular ENS, is determined for all simulated scenarios.

$\sum_{e \in i^{+}} f_{e}^{t}-\sum_{e \in i^{-}} f_{e}^{t}+\sum_{g \in G(i)} P_{g}^{t}=d_{i}^{t}+E N S_{i}^{t}, \forall i \in V, \forall t \in T$

According to (2), every node $i \in V$ should satisfy for all hours $t \in T$ a power balance equation, where the sum of the demand, $-d_{i}^{t}$, the generation, $\sum_{g \in G(i)} P_{g}^{t}$, and the flows, $\sum_{e \in i^{+}} f_{e}^{t}-\sum_{e \in i^{-}} f_{e}^{t}$ should be equal to zero, unless the (slack variable) $E N S_{i}^{t}$ is positive. The two aforementioned operation models present the objective function shown in (3).

$$
\sum_{t \in T} \sum_{g \in G}\left(P_{g}^{t} c p_{g}+v u_{g}^{t} c u_{g}+v d_{g}^{t} c d_{g}\right)+\sum_{i \in V} E N S_{i}^{t} c_{e n s}
$$

The objective function (3) is divided into the cost of operation and the cost of unsupplied energy, where $c p_{g}, c u_{g}$, and $c d_{g}$ are the production, start-up, and shut-down costs, respectively $\left(P_{g}^{t}\right.$ are continuous variables and $v u_{g}^{t}, v d_{g}^{t}$ are binary variables). $E N S_{i}^{t}$ is the unsupplied energy of node $i \in V$, and $c_{e n s}$ is the unit cost of the energy not supplied, i.e. the value of lost load (VoLL). The $E N S_{i}^{t}$ variables are relevant in the post-contingency dispatch model $\left(E N S_{i}^{t}=0\right.$ in the unit commitment model). Note that we do not simulate the effects that an earthquake has on the demand for energy. While our framework can address this by including local distribution network degradation in the Monte Carlo simulations that construct scenarios, a detailed study of the impact of earthquakes on local distribution systems is beyond the scope of this paper. It is assumed that maintaining the energy demand after an earthquake creates a conservative scenario since this makes the amount of ENS due to transmission network infrastructure failure caused by an earthquake as large as possible, and where such ENS is only due to transmission failures that can be addressed with the investment decisions under consideration.

It is important to mention that the previous models are run within a sequential Monte Carlo framework where the natural hazard occurred originates a set of outage scenarios whose consequences are determined via sequential Monte Carlo simulations to capture the detailed (e.g. hourly) system response and restoration. Within this Monte Carlo simulation, not only realizations of outages are determined, but also the repair times of outaged network components, whose distribution function is exponential with a known rate. In those hours without an earthquake, outage rates are those under normal conditions and thus (further) network contingencies may still occur. Fig. 5 shows how the UC and the PCD models are run in a case in which there are outages at two different times, where the first one is caused by a major earthquake. 


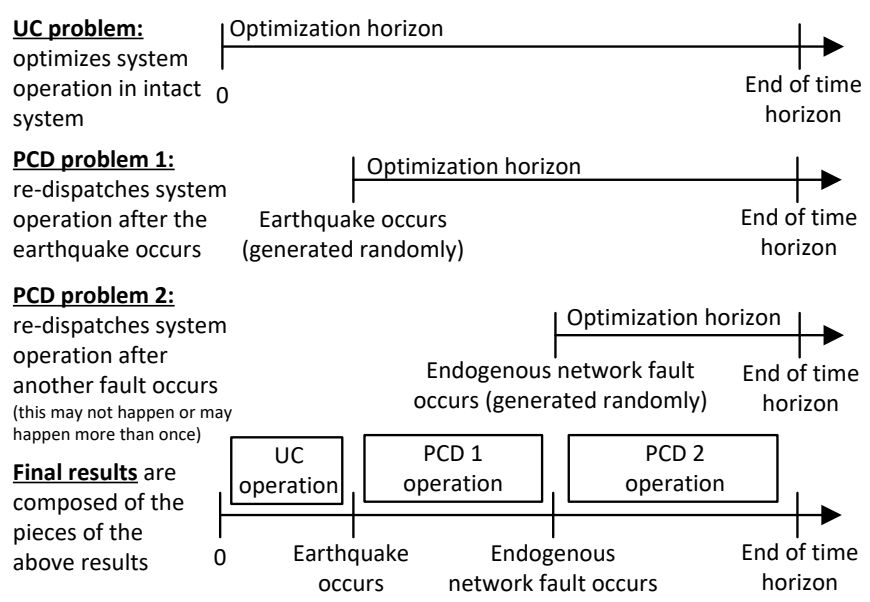

Fig. 5. Interactions between UC and PCD models and their respective optimization time horizons. Resulting generation outputs in a model define the initial conditions for the consecutive model.

\section{The optimizer}

We propose an optimization procedure that considers the output of the simulation as a general function $\mu(x)$ with unknown structure that, in our case, quantifies the resilience level (or its inverse) of a given power network that we want to optimize. A general OvS formulation is shown in (4).

$$
\min _{x \in \Theta} \mu(x) .
$$

The set $\Theta$ has a finite number of feasible solutions, and $\mu(x)$ is a black-box function, i.e. we can only estimate $\mu(x)$ numerically via sampling. For a brief introduction to OvS see [34].

Various metrics have been introduced lately to quantify power system resilience [12], [13]. Here, we follow the general principles in [13], which explains that resilient improvements can be understood as limiting exposure to HILP events that are usually placed in the (right) "tail" of the probability density function of ENS, where ENS values are highest. To mathematically represent that tail, we could use various risk metrics such as Conditional Value at Risk (CVaR) or any conditional expectation value of ENS over those scenarios composing the tail. In this context, we assume that increasing network resilience against earthquakes can be directly translated into a minimization of the conditional expectation of the energy not supplied resulting as a consequence of major earthquakes. Such minimization problem will need to feature a budget constraint that contributes to reflecting planner's risk aversion (i.e. the higher the budget, the higher the aversion and willingness to invest in resilient enhancements against HILP $^{3}$ ). Consequently, the model can be written as shown in (5).

$$
\begin{array}{cl}
\min _{x} & \left\{\mathbb{E}_{\xi}[E N S(x, \xi)]\right\} \\
s . t . & \sum_{i \in Q} a_{i} x_{i} \leq b, \quad \forall i \in Q . \\
& x_{i} \in\{0,1\}, \quad \forall i \in
\end{array}
$$

\footnotetext{
${ }^{3}$ Note that as HILP events are extremely rare, these might not present a significant impact on marginal outage probabilities or on the average/expected value of ENS across all system conditions. Consequently, we need to optimize a risk rather than an average metric and thus a level of risk aversion has to be defined to invest in resilience (for a more comprehensive discussion on this topic, please see [13]), which, in this paper, is represented through a budget constraint.
}

Here binary variables $x_{i}$ represent all network enhancement decisions, such as adding new lines, anchoring/hardening substations, etc. Parameters $a_{i}$ represent costs associated with implementation of $x_{i}$ and $b$ is the total budget allowed to be spent in improving system resilience. The set $Q$ contains all possible network enhancement propositions and $\xi$ corresponds to the realization of uncertainty, in this case, system failures due to earthquakes. As failures are originated by the simulated earthquakes, we use the conditional values of failure probabilities obtained from fragility curves. Consequently, a key feature of this approach is that the allowed budget $b$ will be used in those network enhancements that feature the largest impacts on minimizing ENS caused by natural hazards. In the Appendix, we provide more details of the algorithm implemented.

We also illustrate the use of other metrics such as those proposed in [12] within our framework in order to improve a specific aspect of resilience, e.g. system restoration rapidity or recovery rate. In our framework, we adopt a probabilistic view of such metrics in order to improve a specific aspect of resilience in terms of the corresponding conditional expectation value calculated over all simulated cases (e.g. the conditional expectation of system recovery rate across HILP scenarios).

Importantly, the ISC is essentially a heuristic method whose result may change in every run due to its probabilistic nature. To deal with this, we propose a two-step strategy to finally determine the best network enhancement proposition. This strategy, firstly, builds a set of good candidate solutions, which can be obtained by running the ISC approach a number of times (e.g. 10). Then, in a second step, we identify the best solution among the aforementioned set of good candidates by performing a large number of evaluations/simulations (e.g. $10,000)$ on each of them.

\section{CASE STUDY APPLICATIONS}

\section{A. Input Data}

We modified the IEEE 14 bus case study described in [35] by changing the vector of generation installed capacities as described in Table I, where we also show the peak demand condition and the number of lines connecting to each bus. For planning purposes, we consider the following 14 candidate network enhancements:

1) New lines: 1-12, 7-9, 2-3, 11-14, 6-13, each of $100 \mathrm{MW}$ capacity;

2) Hardening buses: 3, 4, 5, 6, 8;

3) New backup distributed generation (DG) (e.g. diesel plants) in buses 3, 4, 5, 6 .

While new lines and backup generation correspond to extra assets that can be added/installed, hardening buses is a decision for strengthening existing infrastructure (in this example, we assume that a bus is actually a substation). Installed capacity of each backup unit is equal to 5 [MW]. Generation (fuel) variable costs are $50,80,150,30$, and $100 \$ / M W h$ for generators in nodes 1, 2, 3, 6 and 8, respectively. For illustration purposes, we consider that the cost of any network enhancement is the same, which is equivalent to have $a_{i}=1$ for all $i$ and thus the budget $b$ becomes an integer value representing the possible number of simultaneous network enhancements that can be carried out. The VoLL is equal to $10 \mathrm{k} \$ / \mathrm{MWh}$. We constrain 
TABLE I

DEMAND, GENERATION AND NETWORK DATA.

\begin{tabular}{|c|c|c|c|}
\hline Bus & Demand [MW] & Generation [MW] & No. of connections \\
\hline 1 & 0 & 100 & 2 \\
2 & 21.7 & 100 & 4 \\
3 & 94.2 & 100 & 2 \\
4 & 47.8 & 0 & 5 \\
5 & 7.6 & 0 & 4 \\
6 & 11.2 & 100 & 4 \\
7 & 0 & 0 & 3 \\
8 & 0 & 100 & 1 \\
9 & 29.5 & 0 & 4 \\
10 & 9 & 0 & 2 \\
11 & 3.5 & 0 & 2 \\
12 & 6.1 & 0 & 2 \\
13 & 13.5 & 0 & 3 \\
14 & 14.9 & 0 & 2 \\
\hline
\end{tabular}

TABLE II

OUTAGE RATES AND RESTORATION TIMES

\begin{tabular}{|c|c|c|c|}
\hline Component & $\begin{array}{c}\text { Failure rate } \\
\text { (marginal) } \\
{[o c c / y r]}\end{array}$ & $\begin{array}{c}\text { Restoration time } \\
\text { (normal) }[\mathrm{h}]\end{array}$ & $\begin{array}{c}\text { Restoration time } \\
\text { (hazard) }[\mathrm{h}]\end{array}$ \\
\hline 100-km line & 2.62 & 28 & 56 \\
Substation & 0.1 & 21 & 42 \\
Generator & 5 & 58 & 116 \\
\hline
\end{tabular}

the total volume of generation reserves to be the size of the largest unit dispatched in every hour. The total simulation time horizon is 5 days equivalent to 120 hours. Outage rates and restoration times of network and generation equipment are those presented in Table II (restoration times under natural hazard conditions are doubled to illustrate the effects of a higher number of requests that the repair crew may face $)^{4}$.

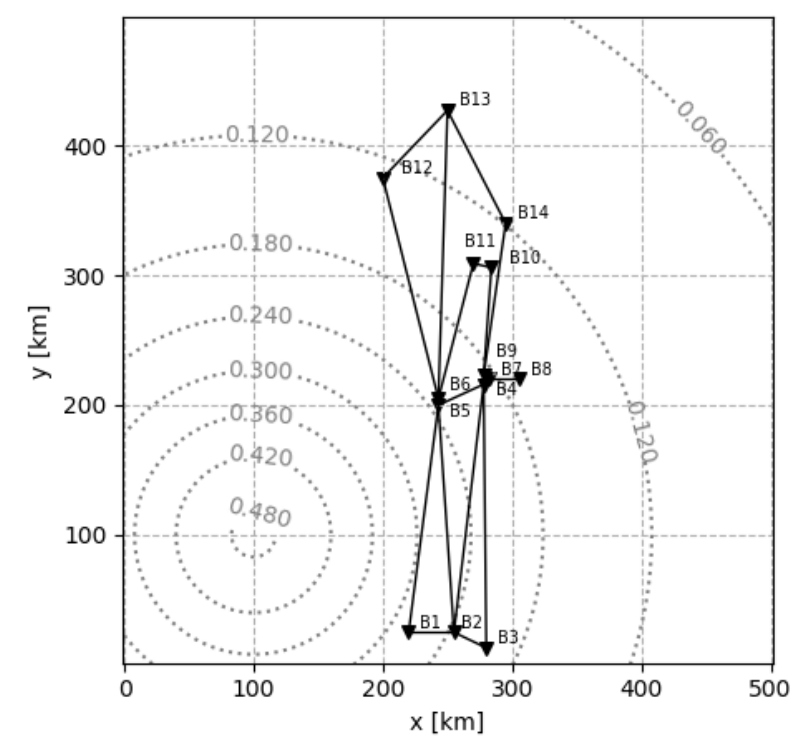

Fig. 6. Network spatial configuration and PGA logarithmic attenuation for an illustrative earthquake with an epicenter at $(100,100) \mathrm{km}$. PGA values (i.e. contours) are shown in $[\mathrm{g}]$.

${ }^{4}$ This assumption in the restoration time has been arbitrarily made in order to reflect the higher restoration time during hazards due to several reasons, such as limited resources of repair crews to respond to multiple damaged assets, in realistic applications, the emergency and restoration procedures of the affected utility should be taken into account to develop an as realistic as possible restoration model.
For the resilience assessment, we use the approach introduced in Section II-B2 including PGA fragility curves from the Hazus report [26] and PGA attenuation model from [32], following equation (1). For the scenario generation, we use the Gutemberg-Richter exponential distribution, ignoring magnitudes below 7.5 Mw. For the earthquakes' location, we use random generation uniformly distributed in the 500x500$\mathrm{km}^{2}$ area illustrated in Fig. 6, which also shows the location of network components and PGA attenuation for a particular earthquake realization. Consequently, outages of network components will result spatially correlated since PGA values are coupled in space, following the logarithmic attenuation in (1). The spatial PGA profile for a given earthquake is illustrated in Fig. 6.

Next, we provide analyses to differentiate the best single network enhancement under two criteria: a reliability criterion and the resilience criterion. Here, we assume that the reliability criterion attempts to minimize ENS as an average value across all outage scenarios that are simulated by using marginal probability values. Under the resilience criterion, instead, the model attempts to minimize ENS as an average value across all outage scenarios originated by earthquakes, simulated by using conditional probability values. In other words, while in reliability we attempt to identify the best investment solution in "average", in resilience we attempt to identify the best investment solution for outages originated by natural hazards, limiting the risk exposure to high impact exogenous events. Next, we also validate the proposed modeling framework to identify resilient network investments by comparing its results against those obtained through complete evaluation of the entire set of network enhancement propositions, which is significantly more costly in computational terms. Finally, we illustrate the results of the framework for identifying (nearly) optimal portfolios of resilient network enhancements among installation of new lines, backup generation and hardened buses by optimizing various resilience metrics.

\section{B. Resilience versus reliability}

Table III shows our results, ranking (at the top) the best network enhancement propositions in terms of both reliability (left) and resilience (right) approaches. In this particular case, we study enhancement propositions that correspond to a single action (i.e. $b=1$ ). Hence, Table III presents, from left to right, the ranked enhancement proposition and its associated EENS and CEENS (the objective function used for reliability is the expected value of ENS (EENS) since we use marginal probability values, and for resilience is the conditional expectation of ENS under HILP events (CEENS); evidently EENS $<$ CEENS). In both cases, reliability and resilience, all network enhancement alternatives of new lines and hardening buses/substations are assessed through 10,000 scenarios, where each represents a random realization of a hazard $\rightarrow$ network outage $\rightarrow$ network repair sequence. In this section, we do not use the ISC model and we do not evaluate the effect of DG. In this case, the proposed assessment is simple since the complete set of feasible solutions presents only 10 elements (5 substation hardening propositions and 5 line propositions), plus the base case with no enhancement. 
The first noticeable insight from these results is the bias towards hardening substations under the resilience approach, in contrast to installation of new transmission lines under the reliability approach. In fact, it is possible to observe from the input data that, in marginal terms, failure rates of lines are significantly larger than failure rates of substation equipment (see Table II). However, given the occurrence of a strong earthquake, this relation switches, due to a disproportional increase in the failure rates of substation equipment with respect to those of transmission towers (due to the fragility curves explained in Section II-B2; derivative of the fragility curve with respect to PGA is much larger for substations than for towers). Interestingly and encouragingly from the perspective of validating the proposed methodology, this is in line with empirical evidence observed in Chile where, for instance, in the 2010 earthquake (of $8.6 \mathrm{Mw}$ of intensity), only $2 \mathrm{~km}$ of transmission lines failed while $25 \%$ of the substations at the transmission level presented some level of damage. It is therefore more efficient to minimize CEENS (conditional to the occurrence of earthquakes) via hardening substations.

In this very particular case, the model identifies that hardening substation 3 (B3) is the most efficient proposition under the resilience approach since it causes the largest decrease in CEENS (from 872.1 to $739.1 \mathrm{MWh}$ ), which is explained because bus 3 is that with the largest energy consumption. Under the reliability approach, instead, the best enhancement proposition corresponds to the installation of line $(1,12)$, which offers a direct connection between generation in bus 1 and a vast consumption area with no generating units in it (buses 9 to 14). Interestingly, the second best alternative under the resilience approach is hardening bus 4 which presents the second largest demand levels along with the highest number of connections with the rest of the system. Clearly, hardening this bus supports both a more resilient supply in bus 4 and in the rest of the system, facilitating transfers in case a natural hazard strikes.

Network investment decisions should consider both resilience and reliability implications. This can be done with a weighted sum of the energy not supplied in reliability $\left(f_{E E N S}(x)\right)$ and resilience $\left(f_{C E E N S}(x)\right)$ objectives. That is, for given weights $\lambda_{1}, \lambda_{2} \geq 0$, consider the objective in (6).

$$
\lambda_{1} f_{E E N S}(x)+\lambda_{2} f_{C E E N S}(x)
$$

This multi-objective function is used to obtain the convex envelope of the Pareto frontier (of efficient/non-dominated solutions) by changing the weights in (6). For the 14-bus instance in this paper, the Pareto frontier is shown as a line in Fig. 7, and corresponds to the convex combination of the objective values of solutions line $(1,12)$ and hardening bus 3 .

It is important to highlight that this example demonstrates the fundamental differences between investment propositions that aim to hedge against average and more risky scenarios.

\section{Validation of $O v S$ model for resilience studies}

In the previous section, our analysis is based on the idea of testing all single network enhancement propositions (i.e. $b=1$ ) and calculate, through 10,000 simulations each, their impacts on CEENS. In that case, the exercise is simple since
TABLE III

RESILIENCE AND RELIABILITY RANKINGS OF SINGLE NETWORK ENHANCEMENT PROPOSITIONS.

\begin{tabular}{|c|c|c|c|}
\hline \multicolumn{2}{|c|}{ Reliability } & \multicolumn{2}{c|}{ Resilience } \\
\hline $\begin{array}{c}\text { Enhancement } \\
\text { proposition }\end{array}$ & $\begin{array}{c}\text { EENS* } \\
{[M W h]}\end{array}$ & $\begin{array}{c}\text { Enhancement } \\
\text { proposition }\end{array}$ & $\begin{array}{c}\text { CEENS* } \\
{[M W h]}\end{array}$ \\
\hline L1,12 & 7.6 & B3 & 739.1 \\
L6,13 & 7.9 & B4 & 785.2 \\
L11,14 & 9.5 & B6 & 803.7 \\
L2,3 & 12.7 & L1,12 & 823 \\
L7,9 & 14.3 & L7,9 & 823.1 \\
B3 & 14.6 & B8 & 830.3 \\
B5 & 14.8 & B5 & 836.6 \\
B8 & 15.2 & L2,3 & 841 \\
B4 & 15.2 & L11,14 & 845.6 \\
B6 & 15.5 & L6,13 & 847.4 \\
Base case & 15.6 & Base case & 872.1 \\
\hline
\end{tabular}

*Evaluated through 10,000 simulations, ensuring

negligible width/error of confidence intervals.

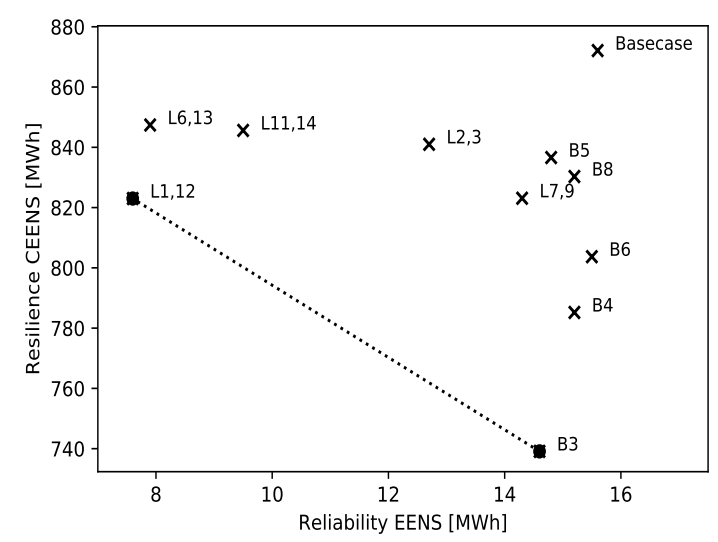

Fig. 7. Resilience and reliability measures of 10 single network enhancement propositions and the base case. Propositions L1,12 and B3 are the only nondominated solutions and characterize the Pareto frontier.

the complete set of feasible solutions is small. In the context of solutions for larger portfolios, though, the idea of calculating CEENS for all possible combinations could be very costly in computational terms. In this particular network, for example, we will need to test $56,176,386,638,848$, and 968 feasible solutions for $b=2,3,4,5,6$ and 7, respectively, considering that for each of them thousands of simulations (e.g. 10,000) are needed to assess their corresponding CEENS. Hence, here we seek to validate our ISC model so as to avoid testing all network enhancement propositions.

In this context, Table IV shows the results of our ISC against those obtained through testing all possible network enhancement propositions, namely, complete enumeration (CE) approach, which runs 10,000 simulations to determine the CEENS associated with each network enhancement proposition. We analyze 3 cases with $b=1,2$ and 3. Our results demonstrated that CE and ICS results are the same for the three budgets. Importantly, while the number of simulations growth significantly under the CE approach (as expected), the ICS needs a much smaller number of iterations to find the (nearly) optimal solution. For example, for $b=3$, we need $1,760,000$ evaluations under the CE approach while only 50,078 evaluation under the ISC (this is the total number of evaluations when ISC is run 10 times). Additionally, we define 
TABLE IV

OvS vS CE

\begin{tabular}{|l|c|c|c|}
\cline { 2 - 4 } \multicolumn{1}{c|}{} & $b=1$ & $b=2$ & $b=3$ \\
\hline Solution & $\mathrm{B} 3$ & $\mathrm{~B} 3 \mathrm{~B} 4$ & $\mathrm{~B} 3 \mathrm{~B} 4 \mathrm{~B} 6$ \\
CEENS* [MWh] & 739.1 & 683.6 & 651.1 \\
No. of feasible solutions & 11 & 56 & 176 \\
No. of CE evaluations & 110,000 & 560,000 & $1,760,000$ \\
No. of ISC evaluations & 10,557 & 20,093 & 50,078 \\
Robustness & 0.6 & 0.9 & 1 \\
\hline
\end{tabular}

*Evaluated through 10,000 simulations, ensuring negligible width/error of confidence intervals.

robustness as the number of times that an ICS solution hits the optimal value (or is, at most, $10 \%$ different from it). Table IV shows that the robustness of the ISC algorithm is very high and, interestingly, increasing with the budget value. In the case $b=3$, for example, 10 out of 10 solutions obtained from the ISC algorithm hit this optimal region. As we always select the best solution out of the best 10 ISC runs, we are able to find the optimal solution for the 3 analyzed budgets. All of the above suggest that ISC offers a robust and practical method to identify resilient network enhancements.

\section{OvS results for larger budgets}

Here we analyze network enhancement solutions with different budgets, in particular, $b=1,2,3,5$ and 7 . Following [13], we also consider the possibility to add backup, distributed generation in specific nodes. This backup generation can only react and generate power in an outage condition and thus cannot interfere in energy trading in the intact system, when no failure occurs.

Table V shows the results for each budget value, while Fig. 8 emphasizes the difference in terms of CEENS improvements between solutions with and without distributed generation.

Interestingly, the patterns to harden buses follows a twofold rationale: (i) securing those buses with large energy consumptions (buses 3 and 4) and (ii) those with a large number of interconnections with other buses (bus 4 and 6). Furthermore, distributed backup generation is placed in order to secure supply for large energy demand volumes (in bus 3 ) in case the main system collapses. Remarkably, if the model is prevented to install backup generation, the effects on CEENS of alternative network enhancement propositions are very limited compared with the improvements driven by backup generation. This suggests that distributed generation (and, in general, distributed energy resources that may be vastly available in the future), may be a more efficient way to improve system-level resilience.

Interestingly, notice that the resilience improvement due to a combination of network enhancements is not equal to the summation of the corresponding individual resilience improvements. For example, the resilience improvement due to hardening B3 only is equal to $133 \mathrm{MWh}(872.1$ - 739.1 MWh in Table III) and due to hardening B4 only is equal to 86.9 MWh (872.1 - 785.2 MWh in Table III). However, the resilience improvement due to the combined effect of B3 and B4 is equal to $188.5 \mathrm{MWh}(872.1-683.6 \mathrm{MWh}$ in Table V), which is different to $133+86.9 \mathrm{MWh}=219.9 \mathrm{MWh}$. This highlights the importance of assessing the effect on system resilience of the entire portfolio of network enhancements, rather than approximating such effect through the individual
TABLE V

OPTIMAL INVESTMENT PORTFOLIOS FOR budget $=0,1,2,3,5,7$.

\begin{tabular}{|c|c|c|c|c|}
\hline & \multicolumn{2}{|c|}{ Without DG } & \multicolumn{2}{|c|}{ With DG } \\
\hline Budget & Solution & $\begin{array}{l}\text { CEENS* } \\
{[M W h]}\end{array}$ & Solution & $\begin{array}{l}\text { CEENS* } \\
{[M W h]}\end{array}$ \\
\hline 0 & Base case & 872.1 & Base case & 872.1 \\
\hline 1 & B3 & 739.1 & B3 & 739.1 \\
\hline 2 & B3 B4 & 683.6 & B3 B4 & 683.6 \\
\hline 3 & B3 B4 B6 & 651.1 & $\begin{array}{l}\text { B3 B4 } \\
\text { 1xDG3 }\end{array}$ & 643.6 \\
\hline 5 & $\begin{array}{l}\text { B3 B4 } \\
\text { B5 B6 } \\
\text { L1, 12 }\end{array}$ & 623.2 & $\begin{array}{c}\text { B3 B4 B6 } \\
2 \text { xDG3 }\end{array}$ & 598.0 \\
\hline 7 & $\begin{array}{c}\text { B3 B4 } \\
\text { B5 B6 B8 } \\
\text { L11,14 L6,13 }\end{array}$ & 616.7 & $\begin{array}{c}\text { B3 B4 B6 } \\
\text { 2xDG3 1xDG4 } \\
\text { 2xDG6 }\end{array}$ & 575.9 \\
\hline
\end{tabular}

*Evaluated through 10,000 simulations, ensuring negligible width/error of confidence intervals.

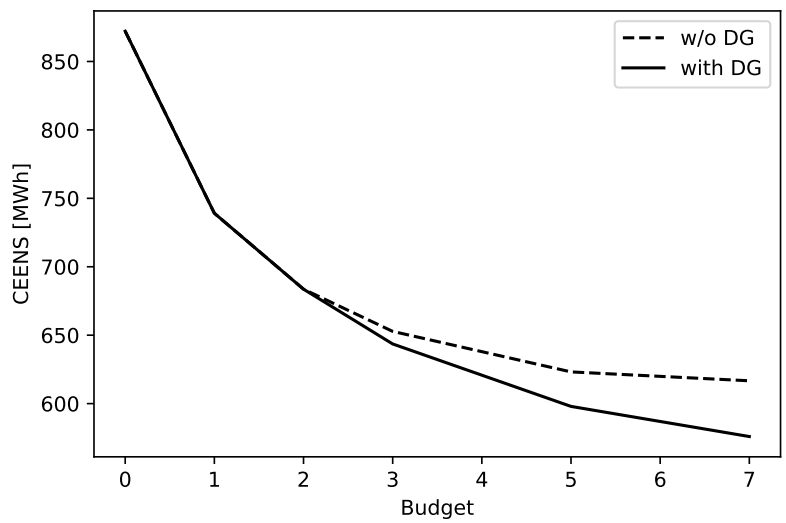

Fig. 8. Conditional expectation of ENS as a function of the budget (with and without DG).

contribution to system resilience of each member of the portfolio. This leads to a large optimization problem since many feasible portfolio combinations must be evaluated. The OvS/ISC addresses this difficulty by avoiding having to evaluate thoroughly every feasible solution. Instead preliminary evaluations, involving few simulations are used to identify likely optimal regions, and additional simulations are used in the local search procedure. Only the likely efficient solutions identified by this procedure are thoroughly evaluated to select the best (see an overview of the algorithm implemented in the Appendix).

\section{E. Optimizing other resilience metrics}

In this section, we show how optimal investment decisions may change if we choose to optimize a different resilience metric. To do so, we still focus on minimizing risks (i.e. minimizing the conditional expectation of a certain metric given a series of earthquakes), but rather than focusing on ENS we select 2 other alternatives. Following the FLEP metrics introduced in [12], we choose:

- To minimize how low supply drops right after the earthquake occurs, so we seek solutions with the minimum demand curtailment (in conditional expected terms) in the first period after the earthquake happens.

- To maximize how promptly demand is reconnected, so we seek solutions with the maximum rate of demand change 
TABLE VI

RANKING OF SINGLE NETWORK ENHANCEMENT PROPOSITIONS OBTAINED BY OPTIMIZING TWO DIFFERENT RESILIENCE METRICS.

\begin{tabular}{|c|c|c|c|}
\hline \multicolumn{2}{|c|}{ Minimizing drop } & \multicolumn{2}{c|}{ Maximizing recovery rate } \\
\hline Solution & $\begin{array}{c}\text { Drop* } \\
{[M W]}\end{array}$ & Solution & $\begin{array}{c}\text { Rate* } \\
{[M W / 120 h]}\end{array}$ \\
\hline B3 & 10.2 & L7,9 & 8.76 \\
B4 & 10.92 & L2,3 & 8.71 \\
B5 & 11.23 & B8 & 8.66 \\
\hline
\end{tabular}

*Evaluated through 10,000 simulations, ensuring negligible width/error of confidence intervals.

(in conditional expected terms) between the first and the last hour of the simulated horizon.

Table VI illustrates how the ranking of the best three investment decisions changes due to the selected resilience metric. While minimizing how low demand drops drives similar solutions to those in Table III, maximizing the demand reconnection rate is biased toward investments in transmission lines. This is so because hardening substations is an attractive solution to avoid disconnections of major demand centers that are directly connected to the hardened substations. This is the case of buses 3 and 4 . In the case of bus 8 , instead, this does not present demand. On the contrary, bus 8 connects one of the five generators to the rest of the system, being an attractive alternative to support a fast system recovery.

\section{CONCLUSIONS}

We propose a framework to determine resilient network enhancements based on a hierarchical approach that, in a first level, proposes network investments and, in a second level, evaluates the improvement in the resilience level associated with the network investment propositions. To do so, we use an OvS approach that is able to deal with a great deal of complexity in the assessment of resilience, including 4 phases: threat characterization, vulnerability of systems components, system response, and system restoration, which are simulated in a sequential Monte Carlo fashion. Further, our approach is able to determine (sequential) evolution of system response and restoration after a hazard occurs considering also decision dependent uncertainty/probabilities.

By using our framework, we distinguished the fundamental differences between reliability- and resilience-driven investments, highlighting the importance of substation-based enhancements in order to successfully face high-impacting earthquakes. Our results also suggest that there is a significant potential support from distributed energy resources in distribution networks, for example, DG that can provide services that increase system-level resilience.

\section{APPENDIX}

The particular Optimization via Simulation (OvS) algorithm utilized in our work is the Industrial Strength COMPASS (ISC) that presents three stages. The first stage is based on Genetic Algorithms (called Niching Genetic Algorithm, NGA), which serves as a global search engine to find good neighborhoods/niches of feasible solutions. The second stage applies the COMPASS algorithm to each of these localminimum structures in order to (locally) improve the solution. We start with the center of the niche yielded by NGA; then we finish when a local minimum with high confidence has been found in every niche. The third stage, the so-called clean-up phase, applies a Ranking and Selection $(\mathrm{R} \& \mathrm{~S})$ procedure to select the best solutions identified in the local phase with a certain probability. The algorithm stops when best solutions selected are compared among each other, discarding poorer solutions and returning the (nearly) optimal set of solutions which are not statistically different according to a given confidence interval that the user can tuned. More details of the ISC algorithm can be found in [28].

Within the above process, the optimizer runs the simulator iteratively for different scenarios. Note that, due to the three uncertainty layers considered by the simulator, a single scenario is composed of a hazard $\rightarrow$ network outage $\rightarrow$ network repair sequence, meaning that Monte Carlo is used to (i) generate a random hazard (e.g. location and magnitude), (ii) generate a possible network outage state, and (iii) generate repair times for the outaged components. Hence, in each iteration a new hazard $\rightarrow$ outage $\rightarrow$ repair scenario is drawn, simulating its pre- and post-contingency operation over a new network enhancement proposition as shown in Fig. 9.

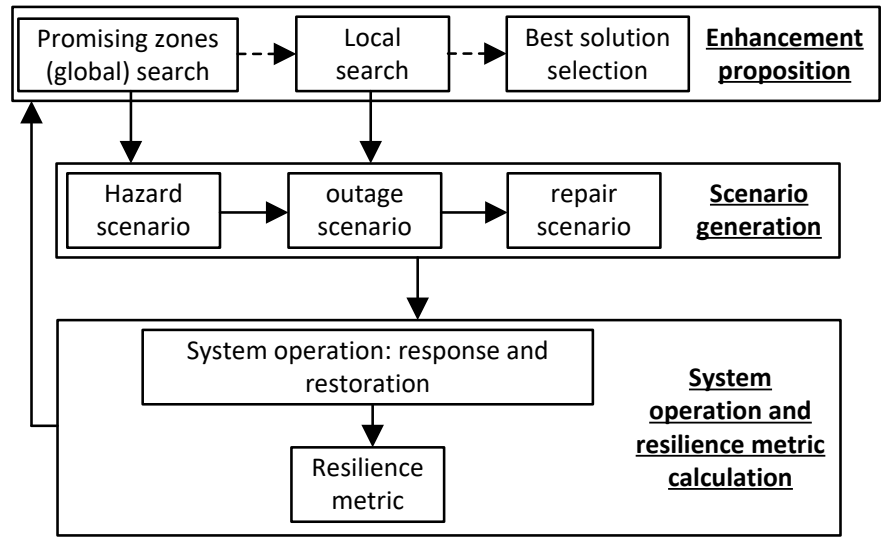

Fig. 9. ISC algorithm applied on the resilient network investment problem. Dashed arrows within the "enhancement proposition" stage indicate that both "local search" and "best solution selection" are run only after "global search" and "local search", respectively, have been run sufficiently many times.

Note that OvS/ISC is computationally intensive as it relies on repeated calls to the simulator, which can include a great deal of complexity and details of system operation. Hence, scaling up this approach to be applied on larger networks may be challenging if the simulator does not run sufficiently fast. Furthermore, larger networks may increase both the computation time of a single simulation and potentially the number of simulations needed (as the solution space of possible network enhancements may increase too). In this vein, there are, at least, two obvious measures to tackle resilience planning studies on larger networks: (i) implementing parallel simulations, taking advantage of distributed computing, and (ii) simplifying the simulations, lowering the levels of detail of system operation (e.g. lowering time resolution). For more comprehensive discussions on the ways and impacts of simplifying assumptions in operation within system expansion planning models, see [36], [37].

\section{ACKNOWLEDGMENT}

The authors gratefully acknowledge the Complex Engineering Systems Institute (CONICYT-PIA-FB0816; ICM P- 
05-004-F), the financial support from Conicyt-Chile (through grants Fondecyt/1181928, Newton-Picarte/MR/N026721/1, SERC Fondap/15110019), and the Powered@NLHPC supercomputing infrastructure (ECM-02).

\section{REFERENCES}

[1] V. D. Gonzalez et al., "Climate change at the idb: Building resilience and reducing emissions," Inter-American Development Bank, Tech. Rep., 2014, accessed Mar. 4, 2015. Available: https://publications.iadb.org/en/publication/16884/climate-changeidb-building-resilience-and-reducing-emissions.

[2] D. A. Ghanem, S. Mander, and C. Gough, "I think we need to get a better generator: Household resilience to disruption to power supply during storm events," Energ. Policy, vol. 92, pp. 171-180, 2016.

[3] Chilean Energy Ministry, "Law number 20.936 (article 87)," https://www.leychile.cl/Navegar?idNorma=1092695, accessed: 2018-0803 .

[4] Y. Zhang, "Earthquake disaster resilience in China," in APEC workshop, Santiago, Chile, 2018

[5] S. Barrientos, "The role of science and technology in earthquake hazard characterization: A Chilean experience," in APEC workshop, Santiago, Chile, 2018.

[6] M. Panteli and P. Mancarella, "Influence of extreme weather and climate change on the resilience of power systems: Impacts and possible mitigation strategies," Electr. Pow. Syst. Res., vol. 127, pp. 259-270, 2015.

[7] Z. Bie, Y. Lin, G. Li, and F. Li, "Battling the extreme: A study on the power system resilience," $P$. IEEE, vol. 105, no. 7, pp. 1253-1266, 2017.

[8] "The definition and quantification of resilience," http://resourcecenter.ieee-pes.org/pes/product/technicalpublications/PESTR0065 04-18, accessed: 2018-08-03.

[9] M. Panteli and P. Mancarella, "The grid: Stronger, bigger, smarter? Presenting a conceptual framework of power system resilience," IEEE Power and Energy Magazine, vol. 13, no. 3, pp. 58-66, 2015.

[10] Y. Wang, C. Chen, J. Wang, and R. Baldick, "Research on resilience of power systems under natural disasters - A review," IEEE T. Power Syst., vol. 31, no. 2, pp. 1604-1613, 2016.

[11] X. Liu et al., "Microgrids for enhancing the power grid resilience in extreme conditions," IEEE T. Smart Grid, vol. 8, no. 2, pp. 589-597, 2017.

[12] M. Panteli, P. Mancarella, D. N. Trakas, E. Kyriakides, and N. D. Hatziargyriou, "Metrics and quantification of operational and infrastructure resilience in power systems," IEEE T. Power Syst., vol. 32, no. 6, pp. 4732-4742, Nov 2017.

[13] G. Strbac, D. Kirschen, and R. Moreno, "Reliability standards for the operation and planning of future electricity networks," Foundations and Trends $\mathbb{R}$ in Electric Energy Systems, vol. 1, no. 3, pp. 143-219, 2016.

[14] S. Espinoza, M. Panteli, P. Mancarella, and H. Rudnick, "Multi-phase assessment and adaptation of power systems resilience to natural hazards," Electr. Pow. Syst. Res., vol. 136, pp. 352-361, 2016.

[15] H. Zhang, H. Yuan, G. Li, and Y. Lin, "Quantitative resilience assessment under a tri-stage framework for power systems," Energies, vol. 11, no. 6, p. 1427, 2018.

[16] M. Panteli, C. Pickering, S. Wilkinson, R. Dawson, and P. Mancarella, "Power system resilience to extreme weather: Fragility modelling, probabilistic impact assessment, and adaptation measures," IEEE T. Power Syst., vol. 32, no. 5, pp. 3747-3757, 2017.

[17] S. Espinoza et al., "Seismic resilience assessment and adaptation of the Northern Chilean power system," in IEEE PES General Meeting, Chicago, United States, 2017.

[18] W. Yuan et al., "Robust optimization-based resilient distribution network planning against natural disasters," IEEE T. Smart Grid, vol. 7, no. 6, pp. 2817-2826, 2016

[19] S. Ma, B. Chen, and Z. Wang, "Resilience enhancement strategy for distribution systems under extreme weather events," IEEE T. Smart Grid, vol. 9, no. 2, pp. 1442-1451, 2018.

[20] N. R. Romero, L. K. Nozick, I. D. Dobson, N. Xu, and D. A. Jones, "Transmission and generation expansion to mitigate seismic risk," IEEE T. Power Syst., vol. 28, no. 4, pp. 3692-3701, 2013.

[21] C. Wang, Y. Hou, F. Qiu, S. Lei, and K. Liu, "Resilience enhancement with sequentially proactive operation strategies," IEEE T. Power Syst., vol. 32, no. 4, pp. 2847-2857, 2017.

[22] D. N. Trakas and N. D. Hatziargyriou, "Optimal distribution system operation for enhancing resilience against wildfires," IEEE T. Power Syst., vol. 33, no. 2, pp. 2260-2271, 2018.
[23] M. Panteli, D. N. Trakas, P. Mancarella, and N. D. Hatziargyriou, "Boosting the power grid resilience to extreme weather events using defensive islanding," IEEE T. Smart Grid, vol. 7, no. 6, pp. 2913-2922, 2016.

[24] N. Romero, N. Xu, L. K. Nozick, I. Dobson, and D. Jones, "Investment planning for electric power systems under terrorist threat," IEEE T. Power Syst., vol. 27, no. 1, pp. 108-116, 2012.

[25] H. Nagarajan, E. Yamangil, R. Bent, P. Van Hentenryck, and S. Backhaus, "Optimal resilient transmission grid design," in Power Systems Computation Conference (PSCC), Genoa, Italy, 2016.

[26] FEMA, Loss Estimation Methodology, MR4-Multi-Hazard, Earthquake Model, Technical Manual, HAZUS report.

[27] R. Billington and R. N. Allan, Reliability evaluation of power systems. Plenum Publishing Corp., New York, NY, 1984.

[28] J. Xu, B. L. Nelson, and J. L. Hong, "Industrial strength compass: A comprehensive algorithm and software for optimization via simulation," ACM T. Model. Comput. S., vol. 20, no. 1, pp. 1-29, 2010.

[29] R. Moreno, D. Pudjianto, and G. Strbac, "Transmission network investment with probabilistic security and corrective control," IEEE T. Power Syst., vol. 28, no. 4, pp. 3935-3944, 2013.

[30] S. M. Burroughs and S. F. Tebbens, "Upper-truncated power laws in natural systems," Pure Appl. Geophys., vol. 158, no. 4, pp. 741-757, 2001.

[31] M. Yoshimoto et al., "Depth-dependent rupture mode along the ecuadorcolombia subduction zone," Geophys. Res. Lett., vol. 44, no. 5, pp. 2203 2210, 2017.

[32] R. L. Boroschek, V. Contreras, D. Y. Kwak, and J. P. Stewart, "Strong ground motion attributes of the $2010 \mathrm{Mw} 8.8$ Maule, Chile, earthquake," Earthquake Spectra, vol. 28, no. S1, pp. S19-S38, 2012.

[33] M. Carrión and J. M. Arroyo, "A computationally efficient mixed-integer linear formulation for the thermal unit commitment problem," IEEE T. Power Syst., vol. 21, no. 3, pp. 1371-1378, 2006.

[34] J. L. Hong and B. L. Nelson, "A brief introduction to optimization via simulation," in Proceedings of the 2009 Winter Simulation Conference (WSC), Austin, United States, 2009.

[35] R. Christie, Power systems test case archive, August 1993, accessed Nov. 4, 2018. Available: http://www.ee.washington.edu/research/pstca/pf14/pg_tca14bus.htm.

[36] A. Flores-Quiroz, R. Palma-Behnke, G. Zakeri, and R. Moreno, "A column generation approach for solving generation expansion planning problems with high renewable energy penetration," Electr. Pow. Syst. Res., vol. 136, pp. 232 - 241, 2016.

[37] R. Moreno, A. Street, J. M. Arroyo, and P. Mancarella, "Planning low-carbon electricity systems under uncertainty considering operational flexibility and smart grid technologies," Phil. Trans. R. Soc. A, vol. 375, no. 2100,2017 